\title{
Primary Neuroendocrine Tumor of the Testis and Osseous, Cardiac, and Lymph Node Metastases in a Young Patient
}

\author{
Matthias Penke \\ Facharztzentrum Lohne, Lohne (Oldenburg), Germany
}

\section{Key Words}

Neuroendocrine tumor · Testis · Somatostatin analogue - Lanreotide · Osseus metastasis .

Cardiac metastasis

\begin{abstract}
A 19-year-old patient was diagnosed with a neuroendocrine tumor (NET) of the testis and suffered from testicular pain and swelling after orchiectomy. After a comprehensive diagnosis, this tumor was shown to be a primary, metastasizing NET of the testis. Primary NETs of the testis are very rare; in the literature, only approximately 60 patients of all ages are mentioned. Less than $20 \%$ of the patients show a metastatic spread. In our patient, lymph node, cardiac, and osseous metastases have been found. It was possible to remove the lymph node metastases through surgery, and the osseous metastases were treated by means of radiation. The exploratory thoracotomy revealed a cardiac metastatic spread; however, it was so extended that a radical resection was not possible. Thereafter, the patient received palliative antiproliferative therapy with the somatostatin analogue lanreotide in the form of monthly subcutaneous injections. This treatment resulted in a stable disease situation. Result: It is possible to administer lanreotide autogel in order to control the growth of the tumor in a young patient with a metastasizing primary NET of the testis with an impossible curative resection of the metastases.

(C) 2014 S. Karger AG, Basel
\end{abstract}

\section{Introduction}

To start with, neuroendocrine tumors (NETs) of the testis are very rare. They only comprise $0.2-1 \%$ of the tumors of the testis [1,2]. Approximately 60 cases have been described 
Penke: Primary Neuroendocrine Tumor of the Testis and Osseous, Cardiac, and Lymph Node Metastases in a Young Patient

in the literature [2,3]. Our own search of the literature published between 1976 and May 2014 resulted in 51 securely identifiable patients with a primary NET of the testis that were published as casuistry or small patient series as well as other, not clearly identifiable, case presentations.

Of the 51 identified patients, 37 were aged between 10 and 83 years with an average age of 48 years (partly calculated as average of a patient series). The individual age was mentioned in 30 of these patients who were divided up into the life decades as follows: 1020 years: 2 patients, $20-30$ years: 5 patients, $30-40$ years: 5 patients, $40-50$ years: 5 patients, $50-60$ years: 5 patients, $60-70$ years: 2 patients, $70-80$ years: 5 patients, and $>80$ years: 1 patient. The largest published series until today with 29 cases [4] reported an average age of 36 years (range 12-65) of patients with a NET of the testis.

Only a small part $(3-13 \%)$ of the primary NETs of the testis is associated with a carcinoid syndrome [1, 4-6]. In the study by Kato et al. [6], out of 15 patients, carcinoid syndrome was present in 2 patients, but serotonin secretion was seen in 10 .

A metastatic spread is reported in $12-20 \%$ of the patients with a primary NET of the testis $[1,3,6,7]$. The size of the tumor of the testis $[3,5,6]$ along with carcinoid syndrome $[3$, 5] have been suspected to be possible risk factors for the metastatic spread. Our own search of the literature revealed metastases in 9 out of 51 patients. The age range indicated for 6 patients with metastases was between 25 and 78 years (average age 59 years). Thus, patients with metastases had a higher average age than patients without metastases (approx. 45 years). Metastases may occur even several years after the orchiectomy due to the primary NET of the testis [8]. In some reports [e.g. 4, 9], the good prognosis of the patients with a primary NET of the testis is mentioned, but this does not apply for the minority of the patients with metastatic spread.

The young patient with a primary NET of the testis presented here is a special case in several aspects.

\section{Case Presentation}

The patient was 19 years old (weight $70 \mathrm{~kg}$, height $180 \mathrm{~cm}$ ) when he reported pain in the right testis and hypogastrium in September 2012. He did not suffer from any other discomfort: neither fever, night sweat, loss of weight nor signs of bleeding or flush symptoms, diarrhea, or vertigo. There was no known history of cancer diseases in his family.

Due to the pain and the clear testicular swelling, a dextral orchiectomy was performed. The histology revealed a NET of the testis without a teratoid tumor with angioinvasion and infiltration of the periorchitic fatty tissue and periorchium. It was possible to detect chromogranin A and synaptophysin in the tumor tissue. The Ki-67 index was $10 \%$ and revealed a histological grading of G2. Serotonin was formed by the tumor tissue. The histological reference result by the Institute of Pathology at the University Clinic of Heidelberg confirmed the diagnosis of a NET of the testis (Ki-67 approx. 5\%).

Based on this result, a comprehensive diagnosis in the tumor staging as well as evidence or exclusion of a primary tumor in other locations was performed between October and December 2012: among others, thoracic/abdominal CT, DOTATOC-PET/CT, and cardiac MRI. The thoracic/abdominal CT resulted in a solitary subpleural consolidation dorsal medial on the left (approx. size $1.7 \mathrm{~cm}$ ), and two suspicious lymph nodes in the epigastrium (approx. size $1.9 \mathrm{~cm}$ ). The DOTATOC-PET/CT revealed the suspected somatostatin receptor-positive lymph node metastases, retroperitoneal para-aortic bilateral osseous metastases in the area of the sacrum (sacral vertebra), as well as an intramyocardial metastasis. The cardiac MRI 
Penke: Primary Neuroendocrine Tumor of the Testis and Osseous, Cardiac, and Lymph Node Metastases in a Young Patient

supported the suspected intramyocardial masses or metastasis $(6 \times 4 \mathrm{~mm})$ in the lateral wall of the left ventricle at the top of the heart.

Our patient was diagnosed with a functional open primary NET of the dextral testis (Ki67: 5-10\%; G2) with serotonin secretion without hormone-induced clinical symptoms. According to the TNM staging for germinal tumors, the NET was classified as follows: pT2 pN1 pM1b. Further, the patient was found to have lymph node metastases (retroperitoneal, para-aortal), osseous metastases (second sacral vertebra on the left), and mediastinal metastatic spread as well as mycardial infiltration.

Due to the patient's young age, the Interdisciplinary Tumor Board decided to implement a maximally intensive therapeutic concept with the target to achieve a long-term remission by a comprehensive tumor debulking. In December 2012, a radical retroperitoneal lymphadenectomy including appendectomy was performed. The histological examination revealed lymph node metastases of the NET of the testis in 2 of the 12 examined interaortocaval lymph nodes in regard to other lymph nodes and the appendix without any tumors.

In January and February 2013, the patient was treated with radiation to the sacrum, with a total dosage of $50 \mathrm{~Gy}$ in 10 fractions, to treat the osseous metastases. In February 2013, the DOTATOC-PET/CT still showed the osseous metastases in the second sacral vertebra and the suspected intramyocardial metastasis in the lateral wall of the left ventricle.

In March 2013, the patient agreed to undergo an exploratory thoracotomy. After prior marking of the tumor with Tc99-D0TATOC, several other myocardial tumor foci with an infiltration of both ventricles were detected under the microscope in addition to the known lesions (PET/CT, cardiac MRI) during the surgery. The intraoperative frozen section analysis revealed NET cells in the biopsies of the suspected metastases. It was not possible to perform a radical curative resection of this comprehensive cardiac metastatic spread.

The patient was informed about the results, and due to the impossible curative treatment and the suspected somatostatin receptor within the metastases, the National Centrum for Tumor Diseases in Heidelberg suggested an antiproliferative therapy with the somatostatin analogue lanreotide (lanreotide autogel; $60 \mathrm{mg}$ every 4 weeks). This therapy was started in April 2013. AS per August 2013, the regular follow-up with the tumor marker DOTATOCPET/CT and cardiac MRI led to a stable disease and wellbeing of the patient.

\section{Discussion}

A 19-year-old patient with testicular pain was diagnosed with a histologically very rare NET of the testis. The comprehensive diagnosis revealed lymph node, cardiac, and (suspected) osseous metastases. It was possible to remove the lymph node metastases through surgery and to radiate the suspected osseous metastases in a fractionated way. An exploratory thoracotomy revealed a comprehensive, not curatively resectable cardiac metastatic spread. The patient received palliative antiproliferative monthly therapy with lanreotide. After 4 months of therapy, a stable disease situation could be observed.

The presented patient is a special case in the scope of this very rare disease under several aspects. In the literature, a maximum of 10 patients under 20 years of age with a primary NET of the testis have been reported. Thus, the patient presented here is one of the very first with a metastasizing primary NET of the testis at such a young age. Even when regarding osseous or cardiac metastases in a primary NET of the testis, only single reports are available. In 1991, metastases in the neck and thoracic vertebrae were reported in a 53year-old Japanese patient [10], and in 1981, cardiac metastases were reported in a 76-yearold US patient [11]. 


\begin{tabular}{l|l}
\hline DOI: $10.1159 / 000369988$ & $\begin{array}{l}\text { C 2014 S. Karger AG, Basel } \\
\text { www.karger.com/cro }\end{array}$ \\
\hline
\end{tabular}

Penke: Primary Neuroendocrine Tumor of the Testis and Osseous, Cardiac, and Lymph Node Metastases in a Young Patient

The most common symptom of primary NETs of the testis is unpainful or almost unpainful testicular swelling; in the case series of Wang et al. [4], this was the case in 15 out of 24 patients with corresponding information. In 6 out of the 24 patients, the tumor was accidentally detected. Due to this very rare primary NET of the testis, a primary tumor resection was required regarding the histological evidence of a NET in the testis $[12,13]$.

A metastasizing NET of the testis that cannot be curatively resected is treated with medication. In this regard, chemotherapy has been proven to be ineffective in most cases, which was was already seen in the case of a 77-year-old Japanese patient with multiple pulmonary and retroperitoneal lymph node metastases in 1997 [14]. In contrast, somatostatin analogues such as octreotide and lanreotide in case of a NET have an antiproliferative effect on the primary tumor and on the metastases. This was also the result of a metaanalysis including 28 trials on the tumor effect of octreotide or lanreotide in case of a NET at different sites [15].

In conclusion, it is possible to administer lanreotide autogel to control the growth of the tumor in a young patient with a metastasizing primary NET of the testis and with impossible surgical treatment of the metastases.

\section{References}

1 Rathert M, Ubrig B, Atkins DJ, Roth S: Carcinoid tumor of the testis (in German). Urologe A 2011;50:340342 .

2 Alsharif S, Al-Shraim M, Alhadi A, et al: Primary neuroendocrine tumor of the testis. Urol Ann 2014;6:173175.

-3 Zavala-Pompa A, Ro JY, el-Naggar A, et al: Primary carcinoid tumor of testis. Immunohistochemical, ultrastructural, and DNA flow cytometric study of three cases with a review of the literature. Cancer 1993;72:1726-1732.

4 Wang WP, Guo C, Berney DM, et al: Primary carcinoid tumors of the testis: a clinicopathologic study of 29 cases. Am J Surg Pathol 2010;34:519-524.

5 Hayashi T, Iida S, Taguchi J, et al: Primary carcinoid of the testis associated with carcinoid syndrome. Int J Urol 2001;8:522-524.

6 Kato N, Motoyama T, Kameda N, et al: Primary carcinoid tumor of the testis: Immunohistochemical, ultrastructural and FISH analysis with review of the literature. Pathol Int 2003;53:680-685.

7 Stroosma OB, Delaere KP: Carcinoid tumours of the testis. BJU Int 2008;101:1101-1105.

8 Sasaki M, Emura M, Kim U, et al: Primary carcinoid tumor of the testis metastatic to the para-aortic lymph nodes in six years after the first operation: a case report (in Japanese). Hinyokika Kiyo 2009;55:233-236.

\$ Han X, Yu L, Yang S, Zheng J: Primary neuroendocrine tumor of the testis: a study of clinicopathological features. Int J Clin Exp Pathol 2014;7:1771-1776.

10 Shimura S, Uchida T, Shitara T, et al: Primary carcinoid tumor of the testis with metastasis to the upper vertebrae. Report of a case (in Japanese). Nihon Hinyokika Gakkai Zasshi 1991;82:1157-1160.

-11 Sullivan JL, Packer JT, Bryant M: Primary malignant carcinoid of the testis. Arch Pathol Lab Med 1981;105:515-517.

12 Chang YH, Chuang CK, Wu CT, et al: Primary carcinoid tumor of the testis: case report. Chang Gung Med J 2002;25:695-699.

13 Hodzic J, Golka K, Schulze H: Primary testicular carcinoid. Med Sci Monit 2004;10:CS46-CS48.

14 Takagi Y, Tanaka J: Primary testicular carcinoid tumor with teratoma: a case report (in Japanese). Hinyokika Kiyo 1997;43:157-159.

15 Sidéris L, Dubé P, Rinke A: Antitumor effects of somatostatin analogs in neuroendocrine tumors. Oncologist 2012;17:747-755. 